\title{
Announcement
}

\section{REVIEWERS FOR VOLUME 14}

In addition to the editorial board, I have been fortunate to have the support of many individuals who have contributed to the review process this past year. I would like to thank them for their time and thoughtful reviews.

Celesta Albonetti

Geoffrey Albert

Susanna Barber

James Bonta

Eugene Borgida

Angela Browne

James Check

Don Conlon

Dewey Cornell

Charles Clark

Jay Casper

Richard Ericson

David Faigman

Robert Felner

Norman J. Finkel

Marc Franklin

Samuel Gaertner

Leslie Goldstein

Edward Gross

Alexander Greer

Robert Hare

Larry Heuer

Ronald F. Holler

Irwin Horowitz

Annmarie Kazyaka

Gerald Koocher
Carol Krafka

Geoffrey Kramer

Rod Lindsay

Dan Linz

Robert Meyer

Reid Meloy

Caton Roberts

Vincent F. Sacco

Regina Schuller

Edward Seidman

Rita Simon

Vicky Smith

Loretta Stalans

Wendy Stock

J. Alexander Tanford

Alan Tomkins

Tom Tyler

Christy Visher

Steven Wasby

C. D. Webster

Richard Wiener

Roselle Wissler

Larry Wrightsman

Barbara Yngvesson

Maria Zaragoza 